\title{
Statistical Analyses of ASE Noise
}

\author{
Mingxiang Gui', Huang Jing² \\ ${ }^{1}$ The First High School of Xiangtan, Xiangtan, China \\ ${ }^{2}$ Physics Department, South China University of Technology, Guangzhou, China \\ Email: Mingxianggui @tom.com, huanggesheng @tom.com
}

How to cite this paper: Gui, M.X. and Jing, H. (2017) Statistical Analyses of ASE Noise. Optics and Photonics Journal, 7, 160-169. https://doi.org/10.4236/opj.2017.710016

Received: September 14, 2017

Accepted: October 21, 2017

Published: October 24, 2017

Copyright (c) 2017 by authors and Scientific Research Publishing Inc. This work is licensed under the Creative Commons Attribution International License (CC BY 4.0).

http://creativecommons.org/licenses/by/4.0/

\begin{abstract}
The evolution of ASE noise and the generation of nonlinear phase shift are analyzed based on the travelling wave solution of ASE noise and its probability density function by solving the Fokker-Planck equation including dispersion effect. Nonlinear effect has strong impact on ASE noise. After the transmission in non-zero dispersion shift fibers + dispersion compensation fibers, due to the nonlinear effect, ASE noise is enhanced. Detailedly, the real part of ASE decreases but the image part increases greatly compared to that with dispersion effect only. Nonlinear phase shift, related to the image part of ASE noise, occurs in this kind of link. The impact of signal intensity on ASE noise induces fluctuations to the both curves of ASE noise and nonlinear phase shift as functions of time, respectively. Furthermore, it results in the non-Gaussian distribution of ASE noise probability density function (side-bands occurring) and brings more than $1 \mathrm{~dB}$ additive BER.
\end{abstract}

\section{Keywords}

Phase Modulation, Parametric Processes

\section{Introduction}

Nonlinear phase noise [1], an additive component of amplified spontaneous emission (ASE) noise induced by the interplay between ASE noise and fiber Kerr nonlinearity, has attracted lots of attention [2] [3] [4] [5] [6]. It is the dominant impairment of differential phase shift keying (DPSK) signals, which successfully suppress the nonlinear fluctuation between signals [7] [8] [9] [10].

From the power spectral density, ASE noise probability density function (p.d.f.) is the Fourier transform of its characteristic function which is determined by the power distribution [2] [3] [4]. The nonlinear phase can be obtained directly and it has been proved that a high dispersion lowers the impact of nonlinear phase [5]-[10]. But usually the nonlinear effect on ASE noise is neglected. Actually, the 
along transmitted signal is so strong and by nonlinear effect, it acts on the ASE noise. So its little change may bring a distinct alteration on ASE noise and counter-act the signal phase again.

In addition, by the power spectral density, the interplay among Kerr nonlinearity, chromatic dispersion and ASE noise in the frequency domain can also be analyzed by the transfer function method [11]. The dependence of nonlinear phase noise and quadrature component of ASE noise is studied in [12], but there are still some unknown effects generating or changing nonlinear phase noise [11] [12] [13].

On the other hand, the p.d.f. of ASE noise can also be calculated by the Fokker-Planck equation [14]. To solve it, the differential around time has to be deleted which means, the dispersion effect is ignored. Even though the dispersion compensation devices will balance most of the dispersion effect, it is no doubt that in the transmission fibers, the complex interaction process that the dispersion acts on nonlinear phase, and produces the intensity fluctuation, then again this fluctuation induces a phase shift and is different from the case of zero dispersion.

To avoid the complex noise probability evolution in fibers, in [5] [6] [10], authors directly calculated the system performance (BER, Q) by the SNR which was the function of power. This is an approach by measuring power to estimate the system performance. Still, the issues on the estimations of ASE noise and nonlinear phase noise are dissolved.

So, in this paper, we attempt to give an inclusive and explicit description about the change of ASE noise and the generation of nonlinear phase noise in dispersion and nonlinear fibers. By my knowledge, this is the first time that in the estimation related to ASE noise, the nonlinear effect is taken into account and it is also the first time that in the p.d.f. of ASE noise the dispersion effect is taken into account.

We start with the transmissions of ASE noise and signals, which satisfy the nonlinear partial differential equations (NLPDE) [11]. Our method is similar to [14], but the in-phase and quadrature components of ASE noise are respectively solved in the time domain. Thus, the dependence between ASE noise in-phase and quadrature components, and their probability density functions impacted by the transmission, are directly derived. This is the base for the practical system bit-error-rate estimation in which ASE noise is impacted by dispersion effect, nonlinear effect and signal formats.

In Section 2, the analytical solution and probability density function of ASE noise are achieved by the travelling wave solution of NLPDE. Based on these solutions, the change of ASE noise originated from dispersion and nonlinear effects is detected for the link of non-zero dispersion shift fibers + dispersion compensation fibers(NZDSFs + DCFs), and nonlinear phase shift caused by nonlinear effect on ASE noise, is analyzed in Section 3. Section 4 presents the conclusion. 


\section{Theory}

In this section, we will derive the nonlinear partial differential equation describing ASE noise in transmission fibers and solve it with the travelling wave method. Subsequently, its probability can been gained based on this solution. Both are the base for the analyses of nonlinear phase noise.

\subsection{Travelling Wave Solution of ASE Noise}

The envelope of electric field satisfies the nonlinear Schrodinger equation:

$$
i \frac{\partial U(z, t)}{\partial z}-\frac{\beta_{2}}{2} \frac{\partial^{2} U(z, t)}{\partial t^{2}}=-\gamma(z) \exp (-2 \alpha z)|U(z, t)|^{2} U(z, t)
$$

where $\beta_{2}$ is the group velocity dispersion, $\gamma$ is the nonlinear coefficient and $\alpha$ is the fiber loss. The field is:

$$
U(z, t)=\sum_{l=1}^{N}\left[u_{l}(z, t)+A_{l}(z, t)\right] \exp \left(-i \omega_{l} t\right)
$$

where $u_{l}(z, t)$ and $A_{l}(z, t)$ are the complex envelopes of signal and ASE noise, respectively. $N$ is the channel number. ASE noise generated in erbium-doped fiber amplifiers (EDFAs) is: $A_{i}(0, t)=A_{I R}(0, t)+i A_{I I}(0, t)$.

$A_{i R}(0, t)$ and $A_{I I}(0, t)$ are statistically real independent stationary white Gaussian processes and $\left\langle A_{I R}(0, t+\tau) A_{i R}^{*}(0, t)\right\rangle=\left\langle A_{I I}(0, t+\tau) A_{I I}^{*}(0, t)\right\rangle=n_{s p} h v_{l}\left(G_{l}-1\right) \Delta v_{l} \delta(\tau)$. In the complete inversion case, $n_{s p}=1 . h$ is the Planck constant. $G_{l}$ is the gain for channel $l$.

Substituting (2) into (1), we can get the equation that $A_{l}(z, t)$ satisfies:

$$
\begin{aligned}
i \frac{\partial A_{l}(z, t)}{\partial z}= & \frac{\beta_{2}}{2}\left(-\omega_{l}^{2}+\frac{\partial^{2}}{\partial t^{2}}-i 2 \omega_{l} \frac{\partial}{\partial t}\right) A_{l}(z, t) \\
& -\gamma(z) \exp (-2 \alpha z)\left|\sum_{j=1}^{N} u_{j}(z, t)+A_{j}(z, t)\right|^{2} A_{l}(z, t)
\end{aligned}
$$

So, the in-phase and quadrature components of ASE noise obey:

$$
\begin{aligned}
\frac{\partial A_{I R}(z, t)}{\partial z}= & -\beta_{2} \omega_{l} \frac{\partial A_{I R}(z, t)}{\partial t}+\frac{1}{2} \beta_{2} \frac{\partial^{2} A_{I I}(z, t)}{\partial t^{2}}-\frac{1}{2} \beta_{2} \omega_{l}^{2} A_{I I} \\
& -\gamma \exp (-2 \alpha z)\left|\sum_{j=1}^{N} u_{j}(z, t)+A_{j}(z, t)\right|^{2} A_{I I} \\
\frac{\partial A_{I I}(z, t)}{\partial z}= & -\beta_{2} \omega_{1} \frac{\partial A_{I I}(z, t)}{\partial t}-\frac{1}{2} \beta_{2} \frac{\partial^{2} A_{I R}(z, t)}{\partial t^{2}}+\frac{1}{2} \beta_{2} \omega_{l}^{2} A_{I R}(z, t) \\
& +\gamma \exp (-2 \alpha z)\left|\sum_{j=1}^{N} u_{j}(z, t)+A_{j}(z, t)\right|^{2} A_{I R}
\end{aligned}
$$

We now seek their travelling wave solution by taking [15] $A_{i R}=\phi(\xi)$ $A_{I I}=\varphi(\xi), \quad \xi=t-c z$. Then, (4) and (5) are converted into:

$$
\phi^{\prime}\left(\beta_{2} \omega_{l}-c\right)=-\left[\frac{1}{2} \beta_{2} \omega_{l}^{2}+\gamma \exp (-2 \alpha z)\left|\sum_{j=1}^{N} u_{j}(z, t)+A_{j}(z, t)\right|^{2}\right] \varphi+\frac{1}{2} \beta_{2} \varphi^{\prime \prime}
$$




$$
\varphi^{\prime}\left(\beta_{2} \omega_{l}-c\right)=\left[\frac{1}{2} \beta_{2} \omega_{l}^{2}+\gamma \exp (-2 \alpha z)\left|\sum_{j=1}^{N} u_{j}(z, t)+A_{j}(z, t)\right|^{2}\right] \phi-\frac{1}{2} \beta_{2} \phi^{\prime \prime}
$$

(7) is differentiated to $\xi$ :

$$
\varphi^{\prime \prime}\left(\beta_{2} \omega_{l}-c\right)=\left[\frac{1}{2} \beta_{2} \omega_{l}^{2}+\gamma \exp (-2 \alpha z)\left|\sum_{j=1}^{N} u_{j}(z, t)+A_{j}(z, t)\right|^{2}\right] \phi^{\prime}-\frac{1}{2} \beta_{2} \phi^{\prime \prime \prime}(8)
$$

Replace $\phi^{\prime}$ and $\phi^{\prime \prime \prime}$ in (8) with (6) and the differential of (6), there are:

$$
\begin{aligned}
\varphi^{\prime \prime}\left(\beta_{2} \omega_{1}-c\right)^{2}= & -\left[\frac{1}{2} \beta_{2} \omega_{l}^{2}+\gamma \exp (-2 \alpha z)\left|\sum_{j=1}^{N} u_{j}(z, t)+A_{j}(z, t)\right|^{2}\right]^{2} \varphi \\
& +\beta_{2}\left[\frac{1}{2} \beta_{2} \omega_{1}^{2}+\gamma \exp (-2 \alpha z)\left|\sum_{j=1}^{N} u_{j}(z, t)+A_{j}(z, t)\right|^{2}\right] \varphi^{\prime \prime}+\frac{1}{4} \beta_{2}^{2} \varphi^{(4)}
\end{aligned}
$$

From (6) and (9), We can easily obtain:

$$
\begin{gathered}
\phi=B\left\{\left[\beta_{2} \omega_{1}^{2} / 2+\gamma \exp (-2 \alpha z)\left|\sum_{j=1}^{N} u_{j}(z, t)+A_{j}(z, t)\right|^{2}\right] \cos k \xi\right. \\
\left.+\beta_{2} k^{2} / 2 \cdot \cos k \xi\right\} /\left(\beta_{2} \omega_{l}-c\right) / k \\
\varphi=B \sin k \xi
\end{gathered}
$$

and

$$
\begin{gathered}
B=A_{l R}(0, t)\left(\beta_{2} \omega_{l}-c\right) k /\left\{\left[\beta_{2} \omega_{l}^{2} / 2+\gamma \exp (-2 \alpha z)\left|\sum_{j=1}^{N} u_{j}(z, t)+A_{j}(z, t)\right|^{2}\right] \cos k t\right. \\
\left.+\beta_{2} k^{2} / 2 \cdot \cos k t\right\} \\
c= \pm\left\{\beta_{2}^{2} k^{2} / 4+\left[\beta_{2} \omega_{l}^{2} / 2+\gamma \exp (-2 \alpha z)^{2}\left|\sum_{j=1}^{N} u_{j}(z, t)+A_{j}(z, t)\right|^{2}\right] / k^{2}\right. \\
+\beta_{2}^{2} \omega_{l}^{2} / 2+\left.\gamma \beta_{2} \exp (-2 \alpha z)\left|\sum_{j=1}^{N} u_{j}(z, t)+A_{j}(z, t)\right|^{2}\right|^{1 / 2}+\beta_{2} \omega_{l} \\
k=\arcsin \left(A_{I I}(0, t) / B\right) / t
\end{gathered}
$$

In the above calculation process, $B, c$ and $k$ should be regarded as constants and $A_{I R}, A_{I I}$ are the functions of the solo variable $\xi$, respectively.

\subsection{Probability Density Function of ASE Noise}

Because $A_{I R}$ and $A_{I I}$ have been solved, the time differentials of (4) and (5) can be calculated. Thus, the stochastic differential equations (Ito forms) around $A_{i R}$ and $A_{I I}$ are:

$$
\frac{\partial A_{i R}(z, t)}{\partial z}=f\left(A_{i R}(z, t)\right)+g\left(A_{i R}(z, t)\right) A_{i R, z=0}
$$




$$
\frac{\partial A_{I I}(z, t)}{\partial z}=f^{\prime}\left(A_{I I}(z, t)\right)+g^{\prime}\left(A_{I I}(z, t)\right) A_{I I, z=0}
$$

where

$$
\begin{aligned}
& f\left(A_{i R}(z, t)\right) \\
& =\beta_{2} k \omega_{1} \sqrt{\left[\frac{\beta_{2} \omega_{l}^{2} / 2+\gamma \exp (-2 \alpha z)\left|\sum_{j=1}^{N} u_{j}(z, t)+A_{j}(z, t)\right|^{2}+\beta_{2} k^{2} / 2}{\left(\beta_{2} \omega_{l}-c\right) k}\right]^{2}-A_{l R}^{2}(z, t)} \\
& g\left(A_{l R}(z, t)\right)=-\frac{\left(\beta_{2} \omega_{1}-c\right) k}{A_{I R, z=0}} \\
& \times \sqrt{\left[\frac{\beta_{2} \omega_{l}^{2} / 2+\gamma \exp (-2 \alpha z)\left|\sum_{j=1}^{N} u_{j}(z, t)+A_{j}(z, t)\right|^{2}+\beta_{2} k^{2} / 2}{\left(\beta_{2} \omega_{l}-c\right) k}\right]^{2}-A_{l R}^{2}(z, t)} \\
& f^{\prime}\left(A_{I I}(z, t)\right)=-\beta_{2} k \omega_{l} \sqrt{B^{2}-A_{I I}^{2}(z, t)} \\
& g^{\prime}\left(A_{I I}(z, t)\right)=\left[B \frac{\beta_{2} \omega_{l}^{2} / 2+\gamma \exp (-2 \alpha z)\left|\sum_{j=1}^{N} u_{j}(z, t)+A_{j}(z, t)\right|^{2}+\beta_{2} k^{2} / 2}{\left(\beta_{2} \omega_{l}-c\right) k}\right]^{2} \\
& \times \frac{\left(\beta_{2} \omega_{1}-c\right) k}{B A_{I I, z=0}} \sqrt{B^{2}-A_{I I}^{2}(z, t)}
\end{aligned}
$$

Now, they can be regarded as the stationary equations and we can gain their probabilities according to Section (7.3) and (7.4) in [16]. By solving the corresponding Fokker-Planck equations of (15) (16), the probabilities of ASE noise are:

$$
\begin{aligned}
& p_{I R}=\frac{C}{\left[g\left(A_{l R}\right)\right]^{2}} \exp \left[2 \int_{-\infty}^{A_{I R}} \frac{f(s)}{[g(s)]^{2}} \mathrm{~d} s\right] \\
& p_{I I}=\frac{C^{\prime}}{\left[g^{\prime}\left(A_{I I}\right)\right]^{2}} \exp \left[2 \int_{-\infty}^{A_{I I}} \frac{f^{\prime}(s)}{\left[g^{\prime}(s)\right]^{2}} \mathrm{~d} s\right]
\end{aligned}
$$

$C, C^{\prime}$ are determined by $\int_{-\infty}^{+\infty} p \mathrm{~d} p=1$. Compared with [13], these probabilities of ASE noise take dispersion effect into account. This is the first time that the p.d.f. of ASE noise simultaneously including dispersion and nonlinear effects is presented.

(21) and (22) are efficient in the models of Gaussian and correlated non-Gaussian processes as our (4) (5). 
Obviously, the Gauss distribution has been distorted. They are no longer symmetrical distributions and both have phase shifts consistent with [13] and as its authors have expected that, "if the dispersion effect was taken into account, the asymmetric modulation side-bands occur." The reasons are that, item $-i \beta_{2} \omega_{1} \frac{\partial}{\partial t} A_{l}(z, t)$ in (3) brings the phase shift, and $\frac{\beta_{2}}{2} \frac{\partial^{2}}{\partial t^{2}} A_{l}(z, t)$ brings the expanding and induces the side bands, the self- and cross-phase modulation effects. Their synthesis impact is amplified by (21), (22) and results in the complete non-Gauss distributions.

So, we have obtained the analytical solution of ASE noise:

$$
a(z, t)=\sum_{l=1}^{N}\left(A_{l R} p_{l R}+i A_{l I} p_{l I}\right) \exp \left(-i \omega_{l} t\right)
$$

\section{Results and Analyses}

In this section, by calculating the signal and ASE noise fields point-to-point, we will simulate the above ASE noise statistical solution and attempt to get some extended results about nonlinear phase noise.

To simplify the problem, we only consider one signal channel. Fiber parameters are listed in Table 1. $u(0, t)=u_{0} \exp \left(\frac{-t^{2}}{2 t_{0}^{2}}\right)$, where $t_{0}$ is the half-width (at $1 / e$ intensity point). $D=-\frac{2 \pi c}{\lambda^{2}} \beta_{2}, u_{0}=5 \mathrm{~mW}$, and $t_{0}=\frac{25}{3} \mathrm{ps}$. The duty ratio is $33 \%$. The bit rate is $2.5 \mathrm{~Gb} / \mathrm{s}$. In the calculation of ASE noise: $\Delta v=50 \mathrm{GHz}$, $G=16.8 \mathrm{~dB} / \mathrm{km}$ and $v=3 \times 10^{8} / 1550 \times 10^{9}(\mathrm{~Hz})$. The link is shown in Figure 1 . We choose the link of NZDSFs + DCFs to show that even though the dispersion effect is quite small, taking it into account is significant.

We have derived the representation of ASE noise in transmission fibers in (23), so the noise evolution can be explicitly shown. We draws ASE noises after they have been transmitted in the link of NZDSFs + DCFs to disclose the nonlinear phase shift induced by nonlinear effect on ASE noise.

In Figure 2, the dots correspond to those only affected by dispersion [12].

$$
\begin{gathered}
a^{\prime}(z, t)=\left(\left\langle\left\{h\left(S_{z}, t+\tau\right) \otimes a(0, t+\tau)\right\}\left\{h\left(S_{z}, t\right) \otimes a(0, t)\right\}^{*}\right\rangle\right)^{\frac{1}{2}}, S_{z}=4 \beta_{2}^{0} z, \\
h\left(S_{z}, t\right)=\frac{1}{\sqrt{2 \pi S_{z}}}\left[\sin \left(\frac{t^{2}}{S_{z}}\right)+\cos \left(\frac{t^{2}}{S_{z}}\right)-i \cos \left(\frac{t^{2}}{S_{z}}\right)+i \sin \left(\frac{t^{2}}{S_{z}}\right)\right], \text { and the solid }
\end{gathered}
$$

lines are based on $(23)\left(\left\langle a(z, t+\tau) a^{*}(z, t)\right\rangle\right)^{1 / 2}$.

Figure 2 plots the $\operatorname{Re}\left(\left\langle a(z, t+\tau) a^{*}(z, t)\right\rangle\right)^{1 / 2}$ and $\operatorname{Im}\left(\left\langle a(z, t+\tau) a^{*}(z, t)\right\rangle\right)^{1 / 2}$ after the transmissions of 1,5 and 10 spans links. The simulations of ASE noise by the power spectral density method are also plotted for the validation of our extending result compared to references [11] [12] [13]. Here, we only calculate the transmission of ASE noise generated in the first amplifier rather than the accumulated value in which each amplifier (every span) generates ASE noise. 
Table 1. Fiber parameters.

\begin{tabular}{cccc}
\hline & $a(\mathrm{~dB} / \mathrm{km})$ & $\gamma(/ \mathrm{km} / \mathrm{W})$ & $D(\mathrm{ps} / \mathrm{nm} / \mathrm{km})$ \\
\hline DCFs & 0.59 & 5.5 & -87 \\
NZDSFs & 0.21 & 2.2 & 4.4 \\
\hline
\end{tabular}

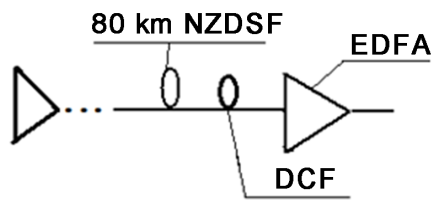

Figure 1. The simulated link.

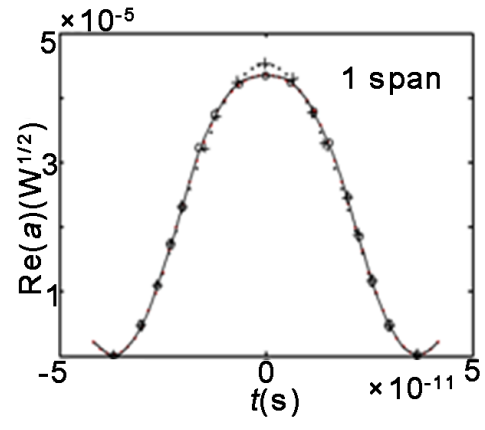

(a)

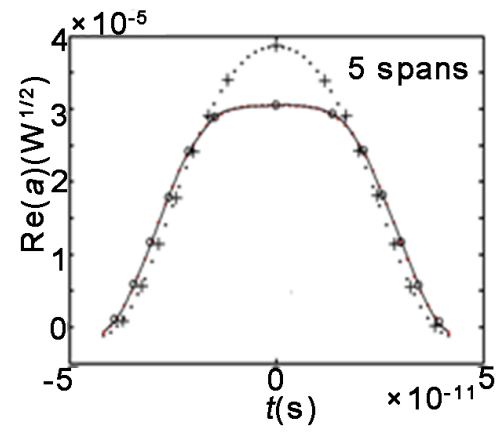

(b)

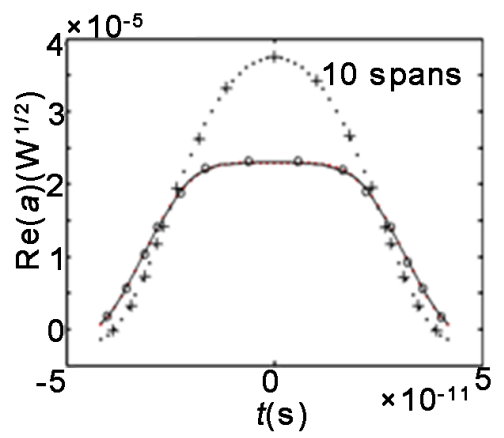

(c)

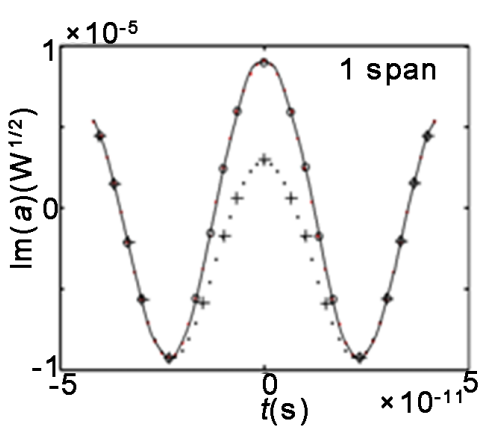

(d)

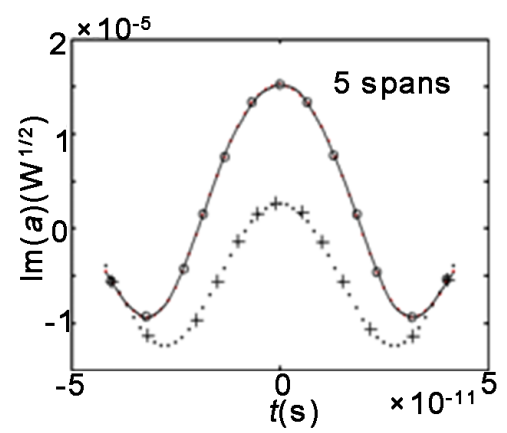

(e)

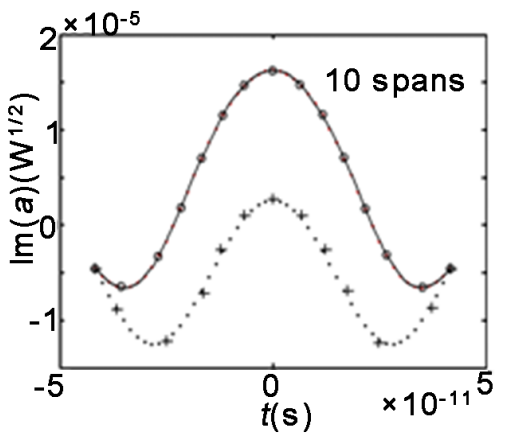

(f)

Figure 2. The evolution of ASE noise after the transmissions of 1,5 and 10 spans NZDSF $(80 \mathrm{~km})+$ DCF $(4.046 \mathrm{~km})$. Marks "o" and “+” are the simulations by the power spectral density method for the cases with and without nonlinear effect, respectively. 
With the increase of distance (span number increases), the deviation between the dotted and the solid lines increases.

Even the dispersion is compensated, due to the nonlinear effect, ASE noise is enhanced. Detailedly, the real part of ASE decreases but the image part increases greatly compared to that with dispersion effect only. The term $-i \beta_{2} \omega_{l} \partial A(z, t) / \partial t$ in (3) brings a phase shift and partly distorts the odd and even symmetries of ASE noise compared with [6] [10] [12]. But this is not explicit in Figure 2 because the transmission is short.

Only the $\operatorname{Re}\left(\left\langle a(z, t+\tau) a^{*}(z, t)\right\rangle\right)^{1 / 2}$ plays roles in the estimation of nonlinear phase noise and according to the calculation formula of [12], fifty percents of the nonlinear phase noise is under-estimated for 10 spans NZDSFs + DCFs if the nonlinear enhancement of ASE noise is not taken into account (Figure 2).

To explicitly exhibit the impact of nonlinear effect on ASE noise, we calculate the bit-error-rate (BER) of a PSK signal as a function of SNR in Figure 3. We calculate BER [3] [16] and SNR [5] [10], separately.

$$
\begin{aligned}
& \mathrm{SNR}=\frac{P_{l}}{N_{\mathrm{ASE}}+N_{\mathrm{NL}}} \\
& \mathrm{BER}=\left.p(\phi)\right|_{\phi=2 \mathrm{rad}}
\end{aligned}
$$

$P_{l}$ is the signal power, $N_{\mathrm{ASE}}$ is the average power of ASE noise and $N_{\mathrm{NL}}$ is the nonlinear fluctuations induced by SPM and XPM. $p(\phi)$ is the p.d.f. as a function of phase. With the decrease of SNR, for the worst case $(\langle\phi\rangle=2 \mathrm{rad}$, the side-band point such as the side band $t=4 \times 10^{-11}(\mathrm{~s})$ in Figure 2(d), the nonlinear effect on ASE noise will bring $1 \mathrm{~dB}$ BER value increase.

We also calculate the phase shift caused by ASE noise [4] in Figure 4.

$$
\sigma=\arctan \frac{\operatorname{Im}(U(z, t))}{\operatorname{Re}(U(z, t))}-\arctan \frac{\operatorname{Im}\left[\int_{-\infty}^{+\infty} u(z, \omega) \exp (-i \omega t) \mathrm{d} \omega\right]}{\operatorname{Re}\left[\int_{-\infty}^{+\infty} u(z, \omega) \exp (-i \omega t) \mathrm{d} \omega\right]}
$$

This phase value is caused by the accumulated ASE noise $\left(\sum^{M}\left(\left\langle a(M z, t+\tau) a^{*}(M z, t)\right\rangle\right)^{1 / 2}, M\right.$ is the span number). The values in Figure 4 are the same order of magnitude as [4] [7] and hence it again verifies our theory. The phase shift caused by ASE noise with nonlinear and dispersion effects is distinct compared to that caused by ASE noise with dispersion only.

There is a trace that a symmetric two-peak spectrum occurs in Figure 2 for quite a short distance (10 spans NZDSFs + DCFs) and this is much clearer in Figure 4. They attribute to that, the signal power is much larger than that of ASE noise and thus brings a faster modulation [17]. We think that, when the distances extend, the asymmetry and fluctuation will be accumulated and there are distinct asymmetry fluctuations in all figures [9].

\section{Conclusion}

In the dispersion and nonlinear fibers, based on the transmission theory of ASE noise and the corresponding Fokker-Planck equations, the change of ASE noise 


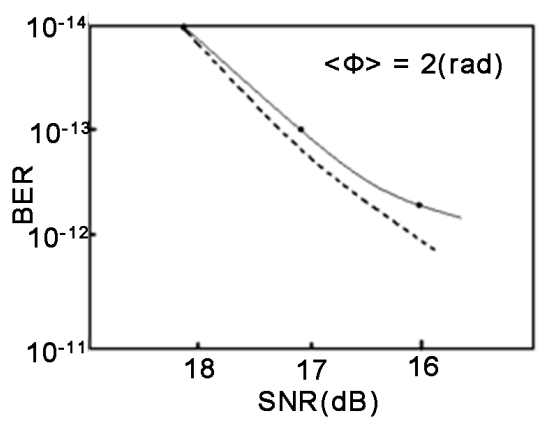

Figure 3. Error probability function of a PSK signal. The black line is our calculation result and the dash line is the case without nonlinear effect on ASE noise.

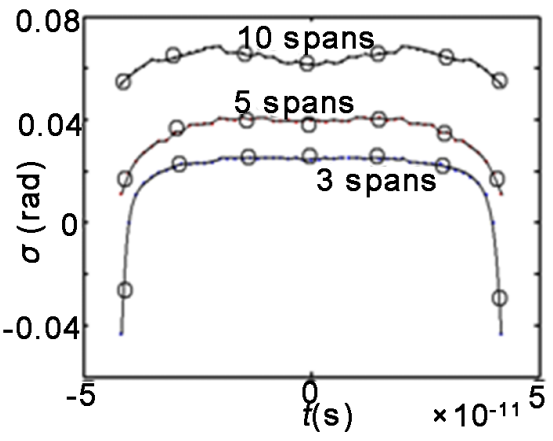

(a)

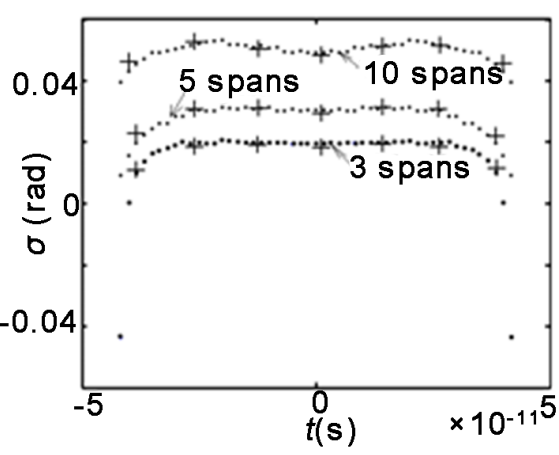

(b)

Figure 4. Phase shift

$$
\sigma=\arctan \frac{\operatorname{Im}(U(z, t))}{\operatorname{Re}(U(z, t))}-\arctan \frac{\operatorname{Im}\left[\int_{-\infty}^{+\infty} u(z, \omega) \exp (-i \omega t) \mathrm{d} \omega\right]}{\operatorname{Re}\left[\int_{-\infty}^{+\infty} u(z, \omega) \exp (-i \omega t) \mathrm{d} \omega\right]} .
$$

ASE noise with nonlinear and dispersion effects; (b) ASE noise with dispersion effect only.

and the generation of nonlinear phase noise can be analyzed. ASE noise is enhanced due to the nonlinear effect. The strong signal not only changes ASE noise probability, statistical amplitude and phase, but also brings an oscillation in the statistical spectrum of nonlinear phase shift.

\section{References}

[1] Gordon, J.P. and Mollenauer, L.F. (1990) Phase Noise in Photonic Communications Systems Using Linear Amplifiers. Optics Letters, 15, 1351-1353. https://doi.org/10.1364/OL.15.001351

[2] Zhu, X.M. and Kumar, S. (2010) Nonlinear Phase Noise in Coherent Optical OFDM Transmission Systems. Optics Express, 18, 7347-7360. https://doi.org/10.1364/OE.18.007347

[3] Mecozzi, A. (2004) Probability Density Functions of the Nonlinear Phase Noise. Optics Letters, 29, 673-675. https://doi.org/10.1364/OL.29.000673

[4] Secondini,M. and Frezzini, M. and Forestieri, E. (2009) Analytical Performance Evaluation of Optical DQPSK Systems With Postdetection Filtering. IEEE Photonics Technology Letters, 21, 908-910. https://doi.org/10.1109/LPT.2009.2020301 
[5] Poggiolini, P. and Bosco, G. and Carena, A. and Curri, V. and Forghieri, F. (2011) A simple and Accurate Model for Non-Linear Propagation Effects in Uncompensated Coherent Transmission Links. 13 th International Conference on Transparent Optical Networks (ICTON), Stockholm, 26-30 June 2011, 1-6.

[6] Vacondio, F. and Rival, O. and Simonneau, C. and Grellier, E. and Bononi, A. and Lorcy, L. and Antona, J.-C. and Bigo, S. (2012) On Nonlinear Distortions of Highly Dispersive Optical Coherent Systems. Optics Express, 20, 1022-1032.

https://doi.org/10.1364/OE.20.001022

[7] Zhang, F., Bunge, C.-A. and Petermann, K. (2006) Analysis of Nonlinear Phase Noise in Single-Channel Return-to Zero Differential Phase-Shift Keying Transmission Systems. Optics Letters, 31, 1038-1040. https://doi.org/10.1364/OL.31.001038

[8] Ho, K.-P. and Wang, H.-C. (2005) Comparison of Nonlinear Phase Noise and Intrachannel Four-Wave Mixing for RZ-DPSK Signals in Dispersive Transmission Systems. IEEE Photonics Technology Letters, 17, 1426-1428. https://doi.org/10.1109/LPT.2005.848542

[9] Ho, K.-P. (2004) Impact of Nonlinear Phase Noise to DPSK Signals: A Comparison of Different Models. IEEE Photonics Technology Letters, 16, 1403-1405. https://doi.org/10.1109/LPT.2004.826054

[10] Grellier, E. and Bononi, A. (2011) Quality Parameter for Coherent Transmissions with Gaussian-Distributed Nonlinear Noise. Optics Express, 19, 12781-12788. https://doi.org/10.1364/OE.19.012781

[11] Demir, A. (2007) Nonlinear Phase Noise in Optical-Fiber-Communication Systems. Journal of Lightwave Technology, 25, 2002-2032. https://doi.org/10.1109/JLT.2007.900888

[12] Ho, K.-P. and Wang, H.-C. (2006) Effect of Dispersion on Nonlinear Phase Noise, Optics Letters, 31, 2109-2111. https://doi.org/10.1364/OL.31.002109

[13] Dlubek, M.P., Phillips, A.J. and Larkins, E.C. (2008) Nonlinear Evolution of Gaussian ASE Noise in ZMNL Fiber. Journal of Lightwave Technology, 26, 891-898. https://doi.org/10.1109/JLT.2008.917373

[14] Serena, P., Orlandin, A. and Bononi, A. (2006) Parametric-Gain Approach to the Analysis of Single-Channel DPSK/DQPSK Systems with Nonlinear Phase Noise. Journal of Lightwave Technology, 24, 2026-2037. https://doi.org/10.1109/JLT.2006.872686

[15] Nichel, J. and Schurmann, H.W. and Serov, V.S. (2005) Some Elliptic Travelling Wave Solution to the Nonikov-Vesela Equation. Proceedings of the International Conference on Days on Diffraction, St. Petersburg, 28 January 2005, 177-186.

[16] Primak, S., Kontorovich, V. and Lyandres, V. (2004) Stochastic Method and Their Applications to Communications: Stochastic Differential Equations Approach. John Wiley \& Sons, Hoboken, Chap. 7. https://doi.org/10.1002/0470021187

[17] Agrawal, G.P. (2001) Nonlinear Fiber Optics. 3rd Edition, Academic Press, Chap. 3. 